\title{
Automatic Segmentation of Acute Leukemia Cells
}

\author{
A.H. Kandil \\ Associate Professor \\ Systems and Biomedical Engineering \\ Department \\ Faculty of Engineering, Cairo University.
}

\author{
O. A. Hassan \\ Systems and Biomedical Engineering \\ Department. \\ High Institutes of Engineering El-Shorouk city
}

\begin{abstract}
The recognition of the acute Leukemia blast cells in colored microscopic images is a challenging task. Segmentation is the essential step for image analysis and image processing. In this paper, an algorithm is presented that consists of panel selection followed by segmentation using K-means clustering then a refinement process. This algorithm was applied on public dataset designed for testing segmentation techniques. The results were compared with two different segmentation techniques developed by other researchers on the same data set. Our algorithm results in a sensitivity of $97.4 \%$ and specificity of $98.1 \%$. The developed algorithm was tested to another dataset of samples extracted from patients in local hospitals. The algorithm results in sensitivity of $100 \%$, Specificity of $99.747 \%$ and accuracy of $99.7617 \%$. The results were approved by expert pathologists.
\end{abstract}

\section{General Terms}

Pattern Recognition, Image processing and segmentation.

\section{Keywords}

Leukemia, segmentation, image enhancement, K-means, and watershed method.

\section{INTRODUCTION}

When the bone marrow generates abnormal white blood cells, they are known as the cancerous blood cells [1] which may be lymphocytic or myelogenous. The cancerous blood cells can be either acute or chronic. In the acute case, the patient gets worse very fast while in the chronic case, the patient gets worse slowly [2]. Leukimea can be classified as follows, Chronic Lymphocytic (CLL), Acute Lymphoblastic (ALL), Chronic Myelogenous (CML), and Acute Myelogenous (AML) [2, 3]. The French-American-British (FAB) classification [2, 4] And the WHO proposal [3, 5] are the two main medical classifiers for Leukimea. It was reported in FAB that the Blast cells in the peripheral blood smear classifies the morphology of Acute Myeloid (AMLs), in seven types (M-1 - M-7) and Acute Lymphoblastic (ALL) in ALL-L1, ALL-L2 and ALL-L3 [2] .

Generally, microscopic investigation of the blood cells is performed manually by hematologists using with a light microscope. It is very tedious, time consuming. In case of analyzing a large number of cells, the visual identification is hard to achieve in reasonable time. Several algorithms and techniques have been developed for the blood cells recognition. Image processing is considered as an important tool for successful automatic diagnosis for both AML and ALL cases. In this work, bone marrow images are presented with heterogeneous staining and pixels features such as color and texture.

The remainder of this paper is organized as follows. Section 2 includes the research background. In section 3 , the proposed segmentation algorithm is described. In section 4 , the results of the algorithm are presented and discussed. Finally, the conclusions are drawn in section 5 .

\section{BACKGROUND}

Segmentation is a basic step in the image analysis, its goal is to partition an image into a set of meaningful patterns easier to analyze. Similarity is a key step towards organizing the image pixels into regions that would correspond to semantically meaningful entities in the scene. There are two forms of segmentation, pixel based image segmentation and region based segmentation. According to Sabino DMU et al. [6], segmentation enables features to be extracted without the inclusion of extraneous material by defining the boundaries of the blood cells. Clustering has been widely used in segmentation of grey level images. Chen Q et al. [7] compared the performances of watershed segmentation for binary images with different distance transforms including Euclidean, City block and Chessboard. They pointed out that combining the watershed and Chessboard distance transform resulted in successful segmentation.

Mohamed MMA et al. [8] studied the normal white blood cell nucleus segmentation algorithm. The proposed algorithm is based on Gram-Schmidt orthogonalization technique. Such a technique enhances the arbitrary selected color of RGB and diminishes the two other colors. They demonstrated the highest contrast of the nucleus. Morphological operations were used to enhance the segmentation. They presented an efficient technique for automatic normal blood cell nuclei segmentation. Assessment of the proposed technique on the blood image set gives $85.4 \%$ accuracy. Eosinophil was found to have the highest segmentation accuracy with $90.1 \%$. Lymphocyte and Basophil have the lowest accuracy with $78.3 \%$ and $78.6 \%$ respectively. Their study was based on the image dataset of 367 color image used with the cell type's distributions.

Trivedi MM, Bezdek JC [9] showed that the color based clustering depends on the selection of color space. The used Fuzzy c-Means clustering algorithm that employs a Pyramid Data Structure (PDS) of Aerial images. This structure allows one to represent the original image at various levels with different resolution. According to Kim K. et al. [10] segmentation techniques as region growing, edge detection, threshold based and pixels clustering are considered as the procedure basis. Blood images are captured from colored CCD camera attached to the microscope. Kim K et al. rely on visual assessment to accomplish the segmentation step. Aimi, Salihah et al., pointed that based on the HSI (Hue, Saturation, and Intensity) colour space image. Colour segmentation based on $\mathrm{S}$ component image for both the Blast and nucleus of AML images. Then, they extracted the $\mathrm{S}$ component information from the enhanced RGB image. Develop the $\mathrm{S}$ component histogram (S-plot) from $\mathrm{S}$ component image to obtain 
threshold value. Apply the thresholding technique using a specific threshold value on $\mathrm{S}$ component image result in successful segmentation of the acute Leukemia images while preserving significant features [11]. Mashor M. showed that thresholding and edge based segmentation for 365 images of stained normal peripheral blood samples were taking by the mean of Gismo-Right technique gives $80.6 \%$ accuracy [12]. Mohammed E et al. [13] presented a method to segment normal and CLL lymphocytes into two parts: nucleus, and cytoplasm using a watershed algorithm and optimal thresholding. Their algorithm resulted in a maximum accuracy of $99.92 \%$ for nucleus segmentation, and $99.85 \%$ maximum accuracy for cell segmentation. In this study, a total of 440 colored images were used.

Prasad AS et al. [14] counted the blood cells by automatic segmentation by threshold, watershed segmentation and chessboard distance measurement on blood stream images that gave minimum counting error obtained $+0.5 \%$ for 200 objects in the dataset contain three color images of blood cells.

Vector Quantization (VQ) was used for image processing and segmentation [15]. The formation of training vector is the basic step for cluster formation [12, 15]. VQ is a mapping function that maps k-dimensional vector space to a finite set $\mathrm{CB}=\{\mathrm{C} 1, \ldots, \mathrm{CN}\}$, where $\mathrm{N}$ is the number of code vectors

The code vector $\mathrm{Ci}=\{\mathrm{Ci} 1, \ldots, \mathrm{Cik}\} ; \mathrm{i}=1,2, \ldots, \mathrm{k}$, represents the codebook [1, 12, 16-18]. H. B. Kekre et al. stated that good codebook is the key to successful VQ [15, 19-20].

Overlapped cells were split by joining concave points using separating lines [21]. Hengen H and Labati RD. [22] retained the shape by eroding and region growing to separate the clumped cells. They showed that automated cells splitting by watershed technique is better for cell counting. Labati RD et al. [23, 24], used morphological operators encoded in the structuring element that depend on object shape and characteristics. Median filters and morphological operations applied for noise removal as suggested by researchers [25]. The application of morphological analysis in separating white blood cells was shown in [8].

Kekre H, Archana B, Galiyal HR [24] Compared between two Vector quantization Algorithms, Linde Buzo and Gray, It is known also as Generalized Lloyd Algorithm (GLA) [24] and Kekre's Proportionate Error [24]. LBG and KPE are tested on

dataset of 115 colored images of acute Leukaemia. This results in a segmentation performance of clustering. For AML, in LBG case, Sensitivity is $60 \%$ and Specificity is $90 \%$. While for KPE are 67\%, 92\% successively. While for ALL, the resulting Sensitivity and Specificity of LBG is 55\%, 90\% successively while for KPE they are $60 \%$ and $92 \%$.

We present this manuscript as a base to build an integrated system. It is based on the accuracy and sensitivity to identify and segment of the Acute Leukemia cells. The consequent ability to build up the system for the classification of ALL cells as will be explained in another manuscript entitled of A Decision Support System for Acute Leukaemia Classification Based on Digital Microscopic Images, Which is submitted for publication [26].

\section{MATERIAL AND METHODS}

A proposed algorithm is shown in Fig. 1.

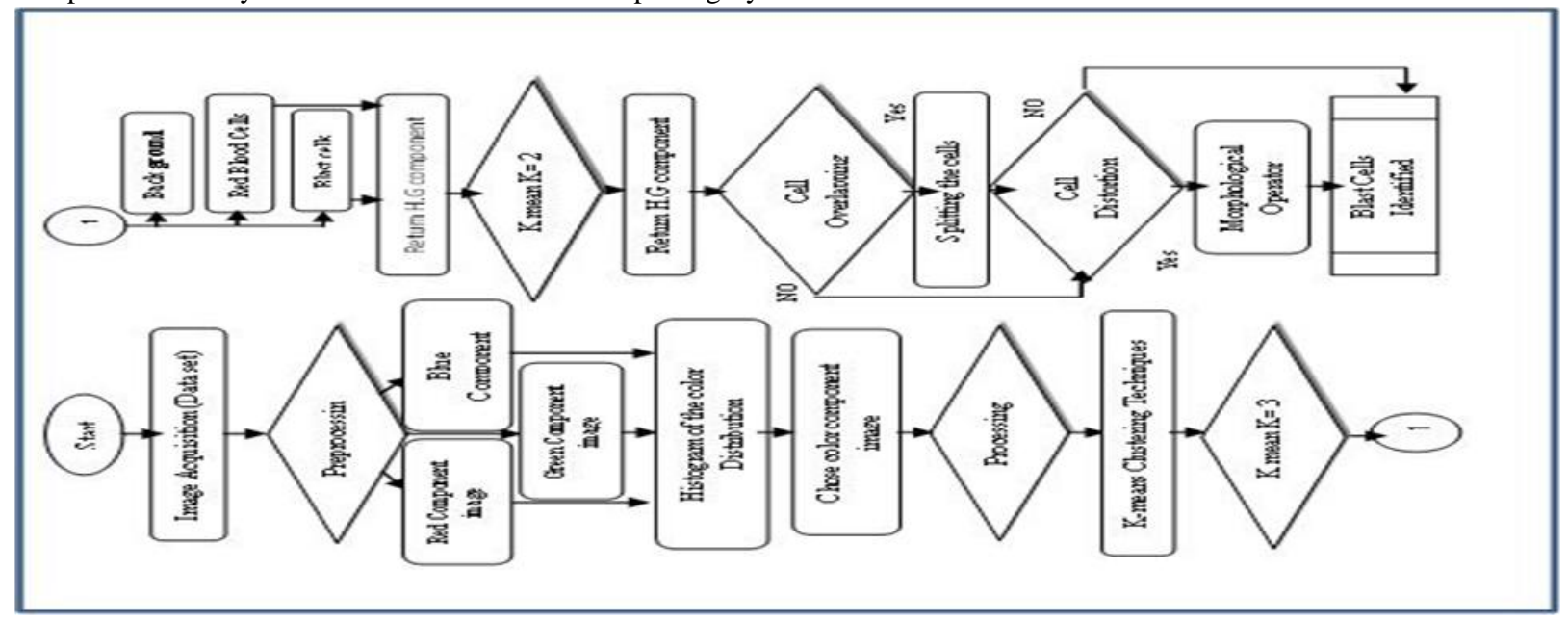

Fig 1: Proposed Flow Chart

\subsection{Material}

The proposed system is applied on two data sets described as follows:

- 115 digital images of size $256 \times 256$ captured with a simple light microscope with three ocular lenses and an analog video CCD which is coupled to a Pinnacle to digitize the captured images [23, 24]. It is a public dataset of microscopic images of blood samples designed for the evaluation and the comparison of algorithms for segmentation and image classification. It was the same material that has been used by Kekre $\mathrm{H}$ [24] and the used database is available [23].

- The second dataset iscollected using light microscope supported by Color video camera digital. The microscope is Olympus BX 40 manufactured in Japan use lens of bower 100X with oil emersion, the camera used is Sony EX wave HAD of Model no.DXC-190. The dataset contains 642 images with size $632 * 480$ pixels classified and recognized by experts. 


\subsection{Method}

\subsubsection{Pre-processing}

The RGB images represented with three color components. They were visually tested as shown in Fig. 2. The histogram of green color distribution and the Green component contains most of the contrast information. This component is chosen for next steps to be processed.

\subsubsection{Processing}

The proposed method is based on the k-means algorithm. It starts by dividing a large set of vectors into groups having the grouping items into $\mathrm{k}$ groups ( $\mathrm{k}$ is the number of pre-chosen groups), minimizing the sum of squared distances between items and the corresponding centroid used in grouping. The procedure consists of the following steps:

$\mathrm{K}$ initial cluster centers Z1(1) ...ZK are chosen. Distribution of samples $\{x\}$ among the $K$ clusters using the relation by the $\mathrm{k}$-th iterative step

$$
\mathrm{X} € \mathrm{Cj}(\mathrm{K}) \text { if }\|\mathrm{X}-\mathrm{Zj}(\mathrm{K})\|<\|\mathrm{X}-\mathrm{Zi}(\mathrm{K})\|
$$

for all $\mathrm{i}=1, \ldots, \mathrm{K} ; \mathrm{i} \mathrm{j}$; where $\mathrm{Cj}(\mathrm{k})$ denotes the set of samples whose cluster centre is $\mathrm{zj}(\mathrm{k})$. New cluster centers $\mathrm{Zj}$ $(\mathrm{k}+1), \mathrm{j}=1,2 \ldots \mathrm{K}$ computed. Minimizing the new cluster according to the sum of the squared distances from all points in $\mathrm{Cj}(\mathrm{k})$. Minimization is measured by the mean of $\mathrm{Cj}(\mathrm{k})$.

$\mathrm{Zj}(\mathrm{K}+1)=1 / \mathrm{Nj}\left[\mathrm{X} \in \mathrm{Cj}(\mathrm{K}) \sum \mathrm{X}, \mathrm{j}=1 \ldots \mathrm{K}\right]$

$\mathrm{Z} \mathrm{j}(\mathrm{K}+1)$ is new cluster centre where $\mathrm{Nj}$ is the number of samples in $\mathrm{Cj}(\mathrm{k})$. If $\mathrm{Zj}(\mathrm{k}+1)=\mathrm{Zj}(\mathrm{k})$ for $\mathrm{j}=1,2 \ldots \mathrm{K}$. The algorithm has finished. Otherwise go to Step 2. The value of $\mathrm{K}$ initial cluster Centers chosen used in clustering are specified as input to the algorithm. K-means clustering algorithm is applied with $\mathrm{k}=3$ and followed by $\mathrm{K}=2$ for segmenting the blast cells. In each step, the returned clustered pixels are the maximum green value.

\subsubsection{Post Processing}

Unwanted region are present in the segmented image. Post processing is required to allocate the blasts only. Image problems such as: Cell Overlapping and Cell Distortion are solved in the enhancement step. A cell separation algorithm is proposed to keep the original shape of the blood cell and to use information of its shape to split the overlapped regions.

Associating pixels into groups is based on the variance using Minimum variance quantization [23]. To identify the overlapped objects, as a single object, leads to errors in measurements and statistics. The watershed distance segmentation is applied to handle this problem. This method automatically splits the overlapped objects. The developed algorithm computes the distance transform for the binary object. Then it complements the distance transform, and force pixels that don't belong to the objects to be $-\infty$. Applying watershed segmentation to the image resulted. The watershed breaks the connected objects at their weakest point, and the separation of overlapped objects in Fig. 3.

Cell distortion and filling object in enhancement step by using morphological operators [24].

Erosion:

$$
A \ominus B=\left\{z \in E \mid B_{\approx} \subseteq A\right\}
$$

Dilation:

$A \oplus B=\bigcup_{b \in B} A_{b}$

Closing:

$A \bullet B=(A \oplus B) \ominus B$

Opening:

$$
A \circ B=(A \ominus B) \oplus B
$$

Where Erosion shrinks or thins the objects in a binary image by the use of structuring element, Dilation is a process that thickens objects in a binary image. Dilation and erosion are used most often and in various combinations. The combination, of these operations, leads to morphological image opening and morphological image closing. Morphological opening can described as an erosion operation followed by a dilation operation. Morphological closing can also described as dilation operation followed by erosion operation [24].

The opening operator is used to fill the missing pixels in the cells according to their similarity with the background color degree. The following Fig. 3 shows the effect of opening operator. The procedures of the developed algorithm are applied to the second data set consisting of 642 images. The results are presented in the next section

\section{RESULTS \& DISCUSSION}

In this work, an algorithm was implemented for successful detection of blast cells. The accuracy of this algorithm was evaluated carefully to estimate its performance in real life. The specificity and sensitivity of this method is calculated using the following formula: [27]. 


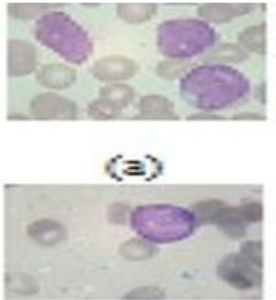

(e)

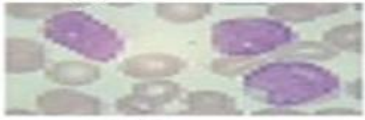

(i)

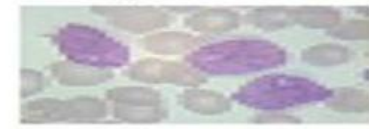

(I)

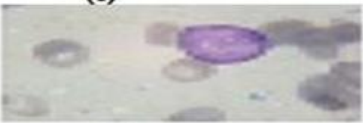

(o)

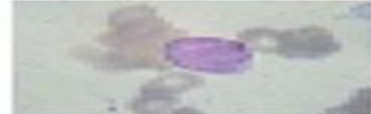

Fig 2

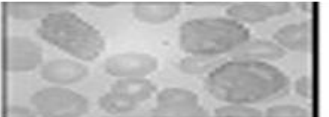

(b)

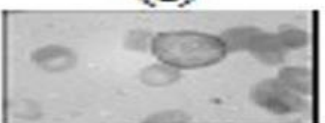

(f)

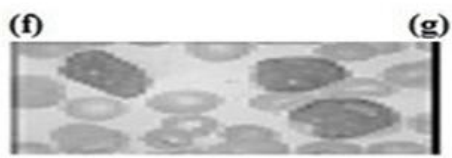

(j)

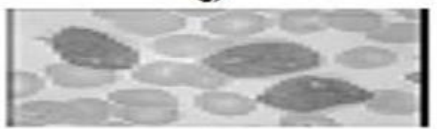

(m)

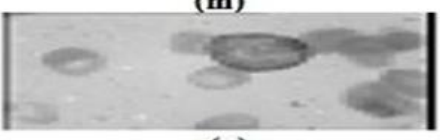

(p)

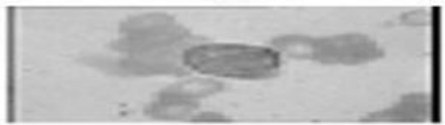

(s)

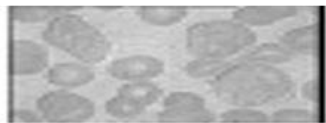

(d)

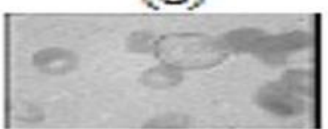

(h)

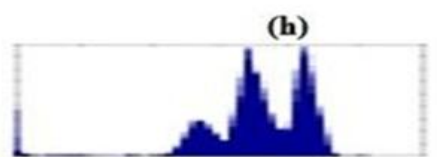

(k)

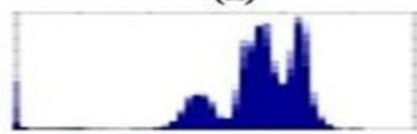

(n)

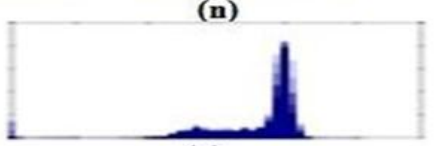

(q)

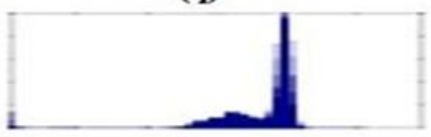

(t)

Sample of color component of the AML (a) Original AML Image, (b) Red color component of image, (c) Green color component of image and (d) Blue color component of image. Sample of color component of ALL images (e) Original ALL Image, (f) Red color component of image, (g) Green color component of image and (h) Blue color component of image. Color image, its green component and green color histogram distribution of AML (i, l) Original AML Image, (j, m) Green panel image and $(k, n)$ Green color histogram. Color image, its green component and green color histogram distribution of ALL (o, r) Original ALL Image, (p, s) Green panel image and (q, t) Green color histogram.

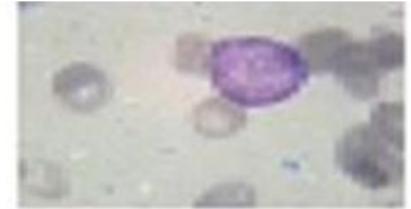

(a)

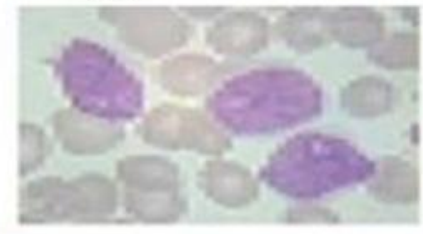

Fig 3 (d)

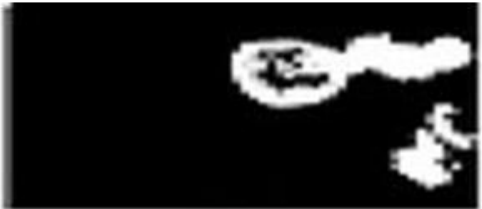

(b)

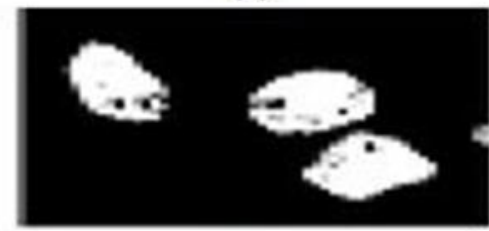

(e)

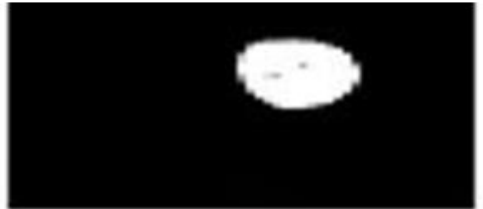

(c)

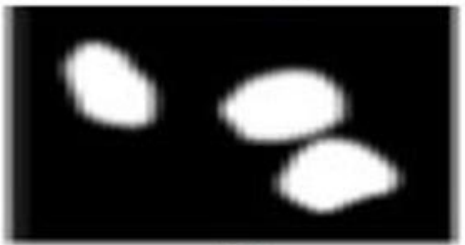

(f)

Fig 3: Cells overlap splitting and extract the cell (a) Original ALL Image, (b) Segmented image with overlapping and (c) Cell extraction; while Segmented images with cell distortion and morphological operator filling gaps (d) Original AML Image, (e) Segmented image with cell distortion and (f) Cell after filling gaps.

Table I proposes a confusion matrix of the algorithm that shows 265 true positive segmented Blast cells. Considering the sensitivity of the algorithm is $97.4 \%$ and specificity $98.1 \%$.

The overall Accuracy calculated by

$=(\mathrm{TN}+\mathrm{TP}) /(\mathrm{TN}+\mathrm{TP}+\mathrm{FN}+\mathrm{FP})=97.4 \%$

And Positive Predictive Value by

$(\mathrm{PPV})=\mathrm{TP} /(\mathrm{TP}+\mathrm{FP})=87.451 \%$.

K-means have sensitivity and specificity of $100 \%$ and $99.1 \%$ in sequence in AML images, and $94.7 \%$ and $96.2 \%$ in sequence of ALL images.

Fig. 4 presents the identified cells in images of AML and ALL after using different Vector Quantization techniques our suggested algorithm using K-means against LBG, KPE applied by Dr. Kekre H June 2013 [24]

Table II, presents a confusion matrix of the algorithm that shows 138 and 127 true positive segmented Blast cells in AML and ALL images in sequence. The collected values were present TP, TN, FP and FN numbers of identified cells in Acute images, AML images and ALL images in Table III 
Considering the sensitivity of the algorithm is $100 \%$ and specificity $99.1 \%$ for AML images and $94.8 \%$ and $96.3 \%$ sensitivity and specificity in sequence of ALL images in Table IV. The sensitivity and specificity comparison in identifying the blast cells in both AML and ALL images with K-means Algorithms against LBG [24] and KPE techniques [24] in the Tables V.

Table 1:Confusion Matrix numbers of Blast cells identifications.

\begin{tabular}{|c|c|c|}
\hline No. Of Images 115 & \multicolumn{2}{|c|}{ Predicated Acute Cells } \\
\hline ctual Stages & TRUE & FALSE \\
\hline POSITIVE & 265 & 38 \\
\hline NEGATIVE & 7 & 2009 \\
\hline overall accuracy & \multicolumn{2}{|c|}{$98.06 \%$} \\
\hline
\end{tabular}

Table 2:Confusion Matrix Numbers Of Blast Cells Identifications In Aml And All Images.

\begin{tabular}{|l|l|l|l|l|}
\hline $\begin{array}{l}\text { No. Of } \\
\text { Images 62 }\end{array}$ & \multicolumn{2}{l|}{$\begin{array}{l}\text { Predicated } \\
\text { AML Cells }\end{array}$} & \multicolumn{2}{l|}{ Predicated } \\
\hline Actual Stages & TRUE & FALSE & TRUE & FALSE \\
\hline POSITIVE & 138 & 12 & 127 & 26 \\
\hline NEGATIVE & 0 & 1337 & 7 & 672 \\
\hline Accuracy & $99.19 \%$ & & $96.03 \%$ & \\
\hline
\end{tabular}

Table 3:Values of TP, TN, FP and FN numbers for Acute images, AML images and ALL images Identified Cells.

\begin{tabular}{|l|l|l|l|l|}
\hline Predicted Stages & TP & TN & FP & FN \\
\hline ACUTE & 265 & 2009 & 38 & 7 \\
\hline AML & 138 & 1337 & 12 & 0 \\
\hline ALL & 127 & 672 & 26 & 7 \\
\hline
\end{tabular}

Table 4:Sensitivity, Specificity and Accuracy percentages.

\begin{tabular}{|c|c|c|c|}
\hline $\begin{array}{c}\text { Predicted } \\
\text { Stages }\end{array}$ & Sensitivity & Specificity & Accuracy \\
\hline ACUTE & 0.974 & 0.981 & $98.06 \%$ \\
\hline AML & 1.000 & 0.991 & $99.19 \%$ \\
\hline ALL & 0.948 & 0.963 & $96.03 \%$ \\
\hline
\end{tabular}

Table 5:Comparing Segmentation Performance Of Identifying Aml, All Type

\begin{tabular}{|l|l|l|l|l|}
\hline & \multicolumn{2}{|l|}{ Identifying AML } & \multicolumn{2}{l|}{ Identifying ALL } \\
\hline Algorithm & Sens. & Spec. & Sens. & Spec. \\
\hline LBG [24] & $60 \%$ & $90 \%$ & $55 \%$ & $90 \%$ \\
\hline KPE [24] & $67 \%$ & $92 \%$ & $60 \%$ & $92 \%$ \\
\hline K-means & $100 \%$ & $99.1 \%$ & $94.8 \%$ & $96.3 \%$ \\
\hline
\end{tabular}

The algorithm is totally successful when applied to second Dataset which consisting of 642images. Its results in detecting the cancer cells and avoiding all unwanted cells from the image in 624 images are with percentage of $97.196 \%$. Table VI shows the confusion matrix of the data observed from the algorithm.

The algorithm results on sensitivity of $1.00 \%$, Specificity of $99.747 \%$ and accuracy of $99.7617 \%$. Since the main objective of this work is identifying the Blast cells objectively rather than subjectively, specific limiting parameters are defined before applying our suggested algorithm effectively. The proposed algorithm applied to well-known public dataset of blood samples organized by Dr. Fabio Scotti, to test their algorithm performances in segmentation and image classification [23]. It was the same material that has been used by Dr. Kekre H June 2013 [24]. The algorithm consists of preprocessing; processing and post processing these main steps were agreed upon among many researchers in the field [24-25, and 28].

Table 6:Confusion Matrix Of Objects In Our Data Set

\begin{tabular}{|c|c|c|}
\hline & \multicolumn{2}{|c|}{ Predicated Acute Cells } \\
\hline Actual Stages & TRUE & FALSE \\
\hline POSITIVE & 802 & 33 \\
\hline NEGATIVE & 0 & 13013 \\
\hline Overall accuracy & \multicolumn{2}{|c|}{$99.7617 \%$} \\
\hline
\end{tabular}

The developed approach is based on the Panel selection, segmentation using K-means clustering, and refinement process succeeds to detect abnormal white blood cells (Blast) [29-31]. Pre-processing is a critical step. That ensures all the following algorithm depends on it. While each researcher chose his way of dealing with the image as Alexander Schmitz et al. with Hodgkin lymphoma images in [28] used Gaussian filtering, application of a threshold for background elimination. Kekre $\mathrm{H}$ [24] used in the same data set in this work, they separated three color planes red, green and blue and used collectively green and blue plane. In this work, the green plane is chosen only depending on the representation showing that the green component contains the highest contrast information in the images.

Segmentation step is the backbone of the algorithm. Vector quantization techniques used for image segmentation. Segmented images by different techniques of vector quantization showed by Kekre $\mathrm{H}$ et al. in [24] LBG, KPE algorithms compared with our suggestion using K-mean clustering. The outcome of segmentation step is represented by specific features that need to be dealt with as: overlapped cells and same color density of cytoplasm and background, not fully connected contour.

Cells splitting can be used to overcome the problem of overlapping using automatic or manual techniques $[14,21$ 22]. Automatic cell splitting is applied using watershed transform followed by morphological operator to overcome overlapping and cell splitting. Succession of identifying blast cells in digital microscopic images after completion algorithm processing is overcoming all problems that have been encountered in the images. 


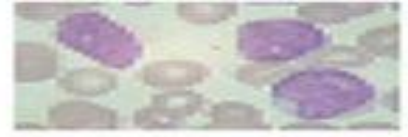

$\Leftrightarrow$

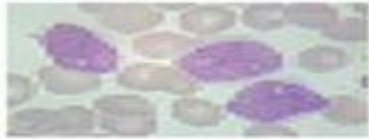

(e)

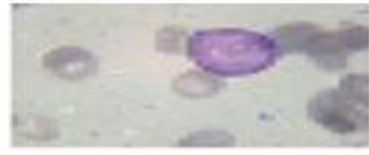

(i)

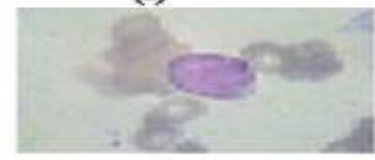

Fig 4

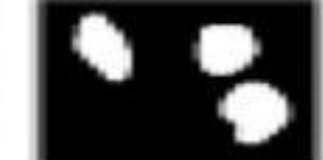

(b)

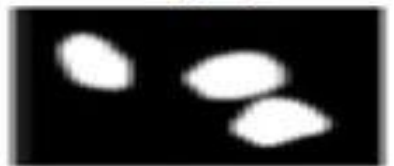

(f)

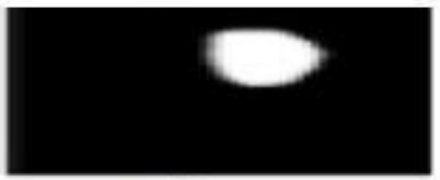

(j)

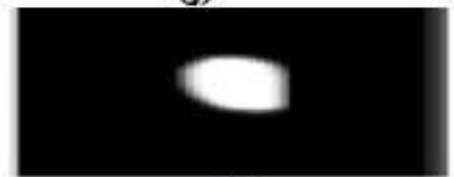

(n)

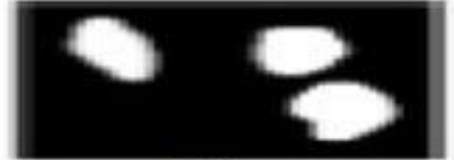

(द)

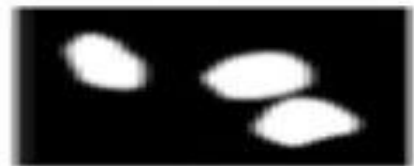

(g)

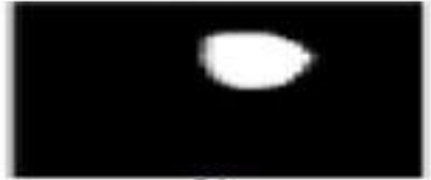

(k)

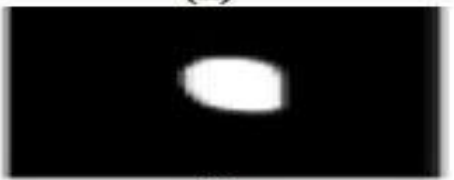

(o)

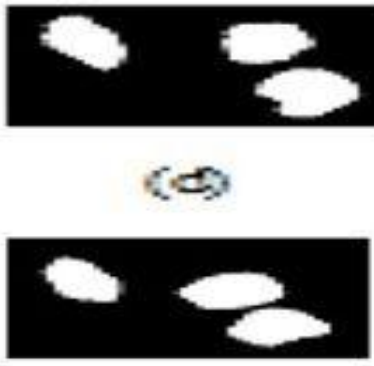

(h)

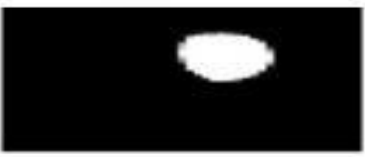

(I)

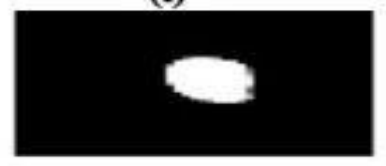

(p)

Fig 4: Different vector quantization algorithms applied on AML images (a, e) Original AML Image, (b, f) Image after applying LBG [24], (c, g) Image after applying KPE [24], (d, h) Image after applying K-means; Different vector quantization algorithms applied o on ALL images (i, m) Original ALL Image, (j, n) Image after applying LBG [24], (k, o) Image after applying KPE [24], (l, p) Image after applying K-means.

The sensitivity and specificity were ensured in this research. Kekre $\mathrm{H}$ et al. found out that their segmentation of blast in acute images with LBG, KPE algorithm with 90\%, 60\% specificity and sensitivity values in sequences [24]. Our Kmeans based algorithm achieved with specificity of $98.1 \%$ and sensitivity of $97.4 \%$.

Specifically, Their results of studying of AML images using LBG the sensitivity and specificity rates $60 \%, 90 \%$ sequentially and KPE rates are 67\%, 92\% sequentially [24]. While, by using K-means in our study, the sensitivity becomes $100 \%$ and specificity rates $99.1 \%$. ALL images using LBG the sensitivity and specificity rates 55\%, 90\% sequentially and KPE rates are $60 \%, 92 \%$ sequentially [24]. While by K-means in our study the sensitivity and specificity rates $94.8 \%$ and $96.3 \%$ sequentially shown in Fig 5 .

Applying the algorithm to the second data set which consists of 642 images with resolution of $632 * 480$ pixels is successful. The segmentation of Acute cells from the images with sensitivity of $100 \%$, Specificity of $99.74 \%$ and Accuracy of $99.76 \%$.

Finally, he algorithm is applied to 757 images from the two datasets; the first consists of 115 images and the second of 642 images. This results in an overall sensitivity of $99.348 \%$, specificity of $99.529 \%$, and accuracy of $99.517 \%$.

\section{CONCLUSION}

Microscopic digital images have been analyzed with the aim of segmentation of the Blast cell. The developed algorithm is able to identify the Blast cells under specified criteria of image processing and enhancement. Packages of image enhancement techniques are applied to improve the image quality. Automatic image segmentation will facilitate the automatic recording of the frequency of the different types of cell.

The comparison between the proposed algorithm using $\mathrm{K}$ means clustering against LBG and KPE for blast detection in acute images were presented. The K-means algorithm shows more accurate results than LBG and KPE.

The success of the proposed system to a dataset used in previous work (115 images) [23], motivated us to test it with another dataset (642 images) this step gives an impressive result demonstrating the accuracy and success of the previously proposed system. Sensitivity is of $100 \%$, Specificity of $99.747 \%$ and accuracy of $99.7617 \%$. This leads to adopting this system in advanced studies using locally developed material for identification and classification of acute myeloid cells. 


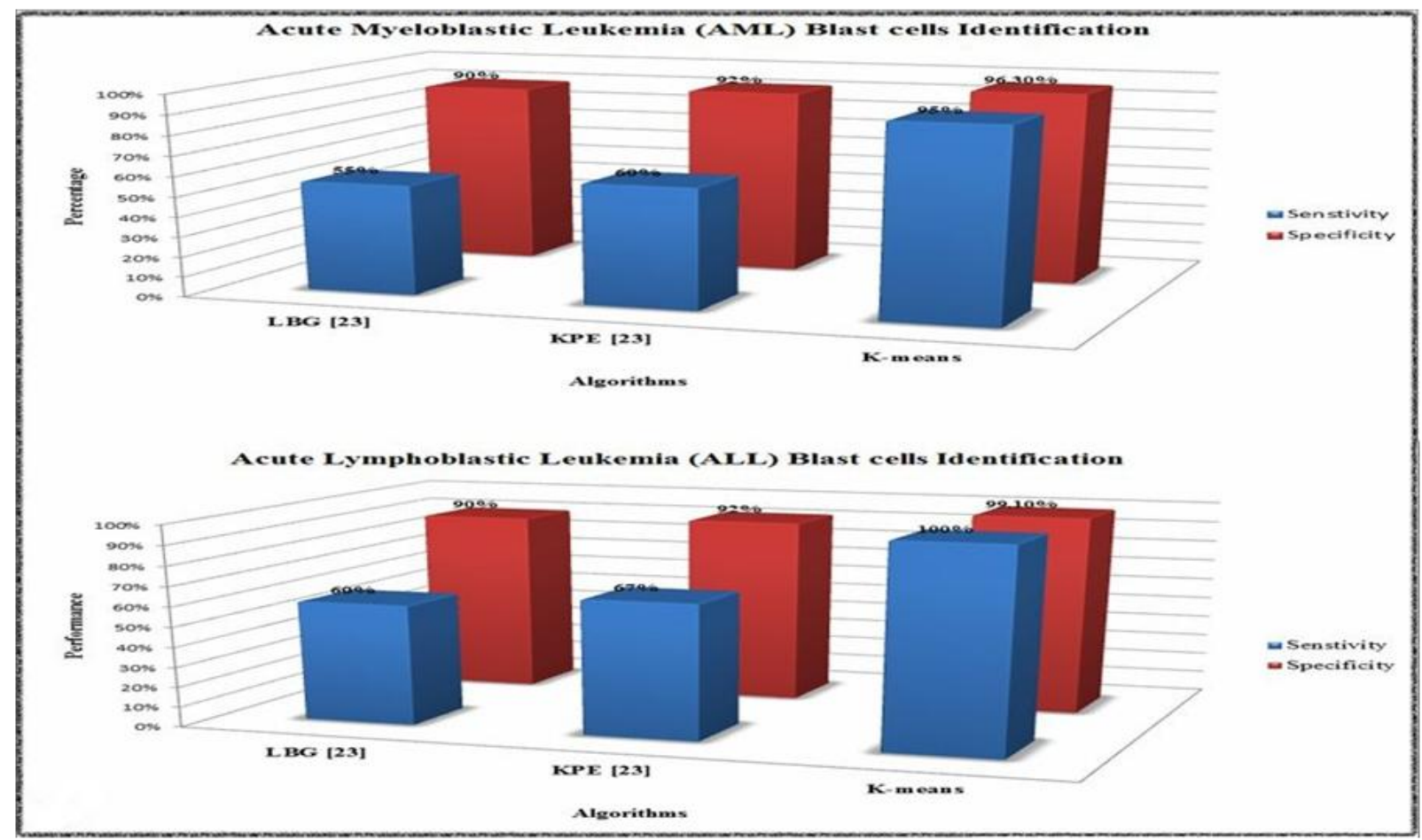

Fig 5: Comparision between three different vector quantization techniques Linde-Buzo-gray (LBG), Kekre's Proportionate Error (KPE) and K-means techniques

\section{ACKNOWLEDGEMENT}

The authors would like to thank Dr. Fabio Scotti Assistant Professor Università degli Studi di Milano Dipartimento di Informatica Via Bramante 65-26013 Crema-Italy for providing public and free dataset of microscopic images of blood samples.

\section{REFERENCES}

[1] Kekre H, Gharge SM, Sarode TK. Tumor Demarcation in Mammography Images using LBG on Probability Image. International Journal of Computer Applications. 2010 June; 3(8): p. 47-53.

[2] Nadia M. Molecular pathology of cancer Cairo: First edition, National cancer institute Cairo University; 1998.

[3] Asaad NY, Dawoud M. Diagnosis and Prognosis of BCell Chronic Lymphocytic Leukemia/Small Lymphocytic Lymphoma (B-CLL/SLL) and Mantle Cell Lymphoma (MCL). J Egypt Natl Canc Inst. 2005 December; 17(4): p. 279-290.

[4] Bennett JM, Catovsky D, Daniel M, Flandrin G, Galton D, Gralnick HR, et al. Proposals for the classification of the acute leukaemias. French-American-British (FAB) co-operative group. British Journal of Haematology. 1976 January 26 ; 33(4): p. 451-458.

[5] Vardiman JW, Thiele J, Arber DA, Brunning RD, Borowitz MJ, Porwit A, et al. The 2008 revision of the World Health Organization (WHO) classification of myeloid neoplasms and acute leukemia: rationale and important changes. Blood. 2009 Jul 30; 114(5): p. $937-$ 951.

[6] Sabino DMU, da Fontoura Costa L, Rizzatti EG, Zago MA. A texture Approach to Leukocyte recognition. Real time Imaging. 2004 OCT; 10(4): p. 205-216.

[7] Chen Q, Yang X, Petriu EM. Watershed Segmentation for Binary Images with Different Distance Transforms. In Haptic, Audio and Visual Environments and Their Applications, 2004. HAVE 2004. Proceedings. The 3rd IEEE International Workshop; 2004; Ottawa, Ontario, Canada. p. 111-116.

[8] Mohamed MMA, Far B. A Fast Technique for White Blood Cells Nuclei Automatic Segmentation Based on Gram-Schmidt Orthogonalization. In IEEE 24th International Conference on Tools with Artificial Intelligence; 2012. p. 947-952.

[9] Trivedi MM, Bezdek JC. Low-level segmentation of aerial images with fuzzy clustering. IEEE Trans. Syst. Man. Cybern. 1986; 16(4): p. 589-598.

[10] Kim K, Jeon J, Choi W, Kim P, Ho YS. Automatic Cell Classification in Human's Peripheral Blood Images Based on Morphological image processing. In AI 2001: Advances in Artificial Intelligence.: Springer; 2001. p. 225-236.

[11] Mashor M, Harun NH, Abdullah AA, Rosline H. Improving Colour Image Segmentation on Acute Myelogenous Leukaemia Images Using Contrast Enhancement Techniques. In IEEE EMBS Conference on Biomedical Engineering \& Sciences (IECBES 2010); 30th November - 2nd December 2010; Kuala Lumpur, Malaysia. p. 246-251.

[12] Mohamed M, Far B. An enhanced threshold based technique for white blood cells nuclei automatic segmentation. In e-Health Networking, Applications and Services (Healthcom), 2012 IEEE 14th International Conference.; 2012; Calgary, Canada. p. 202-207.

[13] Mohammed E, Mohamed MM, Naugler C, Far BH. Chronic lymphocytic leukemia cell segmentation from microscopic blood images using watershed algorithm and optimal thresholding. In 26th IEEE Canadian Conference 
Of Electrical And Computer Engineering (CCECE); 2013; Canada. p. 1-5.

[14] Prasad AS, Latha KS, Rao SK. Separation and counting of blood cells using geometrical features and distance transformed watershed. International Journal of Engineering and Innovative Technology (IJEIT). 2013 August; 3(2).

[15] Kekre H, Thepade SD, Sarode TK, Suryawanshi V. Image Retrieval using Texture Features extracted from GLCM, LBG and KPE. International Journal of Computer Theory and Engineering. 2010 October; 2(5): p. 1793-8201.

[16] Kekre H, Sarode TK, Raul B. Color Image Segmentation using Kekre's Algorithm for Vector Quantization. International Journal of Computer Science (IJCS). 2008 jan; 3(4): p. 287-292.

[17] Kekre H, Sarode TK, Raul B. Color Image Segmentation using Vector Quantization Techniques Based on Energy Ordering Concept. International Journal of Computing Science and Communication Technologies (IJCSCT). 2009 January; 1(2): p. 164-171.

[18] Kekre H, Sarode T. Two Level Vector Quantization Method for Codebook Generation using Kekre's Proportionate Error Algorithm. International Journal of Image Processing. 2010; 4(1): p. 1-10.

[19] Kekre H, Sarode TK. New Clustering Algorithm for Vector Quantization using Rotation of Error Vector. (IJCSIS) International Journal of Computer Science and Information Security. 2010; 7(3): p. 159-165.

[20] Kekre H, Gharge SM, Sarode TK. Image Segmentation of Mammographic Images Using Kekre'S Proportionate Error Technique on Probability Images. International Journal of Computer and Electrical Engineering. 2010 December; 2(6): p. 1048-1052.

[21] Wang W, Song H. Cell Cluster Image Segmentation on Form Analysis. In IEEE Third International Conference on Natural Computation, ICNC; 2007. p. 833-836.

[22] Hengen H, Spoor SL, Pandit MC. Analysis of Blood and Bone Marrow Smears Using Digital Image Processing Techniques. In International Society for Optics and Photonics; 2002. p. 624-635.
[23] Labati RD, Piuri V, Scotti F. The Acute Lymphoblastic Leukemia Image Database For Image Processing. In 18th IEEE international conference on Image processing (ICIP); 2011; Università degli Studi di Milano, Department of Information Technology, via Bramante65, 26013 Crema, Italy. p. 2089-2092.

[24] Kekre H, Archana B, Galiyal HR. Segmentation of Blast using Vector Quantization Technique. International journal of Computer Applications. 2013 June; 4(5).

[25] Soltanzadeh R, Rabbani H, Talebi A. Extraction of nucleolus candidate zone in white blood cells of peripheral blood smear images using curvelet transform. Computational and Mathematical Methods in Medicine. 2012 February; 2012(12,): p. 1-12.

[26] Negm AS, Hassana OA, kandil AH. A Decision Support System for Acute Leukemia Classification from Digital Microscopic Images. Journal of Advanced Research. 2015.

[27] Sharma D, Yadav U, Sharma P. The concept of sensitivity and specificity in relation to two types of errors and its application in medical research. Journal of Reliability and Statistical Studies. 2009; 2(2): p. 53-58.

[28] Schmitz A, Schäfer T, Schäfer H, Döring C, Ackermann J, Dichter N, et al. Automated Image Analysis of Hodgkin lymphoma. 2012 September.

[29] Aimi Salihah A, Mashor MY, Harun NH, Rosline H. Colour Image Enhancement Techniques for Acute Leukemia Blood Cell Morphological Features. In IEEE International Conference on Systems Man and Cybernetics (SMC); 2010. p. 3677-3682.

[30] Aimi Salihah AN, Mashor M, Abdullah AA. Improving Blast Segmentation of Acute Myelogenous Leukemia (AML) Images Using Bright Stretching Technique. In Proceedings of the International Postgraduate Conference on Engineering (IPCE 2010); 2010.; Perlis, Malaysia. p. 16-17.

[31] Halim NA, Mashor M, Abdul Nasir A, Mokhtar N, Rosline H. Nucleus Segmentation Technique for Acute Leukemia. In IEEE 7th International Colloquium on Signal Processing and its Applications, (CSPA); 2011. p. 192-197. 